\title{
RELATIVISTIC RISE OF THE MOST PROBABLE ENERGY LOSS IN A GAS PROPORTIONAL COUNTER*
}

\author{
P. V. RAMANA MURTHY ${ }^{\dagger}$ \\ Department of Physics, Unitersity of Michigan, Ann Arbor, Michigan, L.S.A.
}

Received 19 March 1968

The most probable energy loss in a gaseous medium, as also in other media, was theoretically predicted by Landau to increase with $p_{/} /\left(m c^{\prime}\right)$, the ratio of momentum to the rest mass of a charged particle. The predicted magnitude of this rise (referred to as relativistic rise) was verified up to $p /(m c) \approx 50$ in gases in the previous experiments. We discussed earlier a method of distinguishing protons from pions in the cosmic radiation at energies $\gtrless 100 \mathrm{GeV}$ based on the relativistic rise of the most probable

\section{Introduction}

In a recent paper ${ }^{1}$ ) we discussed the feasibility of distinguishing protons from pions in the cosmic radiation at energies $\gtrsim 100 \mathrm{GeV}$ by using an array of gas proportional counters to measure the relativistic rise of ionization loss and a magnet spectrograph or a total absorption spectrometer to measure the total energy of the incident particle. In that discussion, we assumed that the most probable energy loss increases with the Lorentz factor, $\gamma$ (= ratio of the total energy of a particle to its rest mass), according to the theory of Landau ${ }^{2-4}$ ) corrected for density effect by Sternheimer ${ }^{5}$ ). We recall that the relativistic increase under consideration is quite small; for example, the expected difference in the most probable energy loss by a proton of $100 \mathrm{GeV}[p /(m c) \approx 100]$ and that by a pion of the same energy $[p /(m c) \approx 700]$ is only $\approx 10^{\circ}$.o in argon. For the method discussed by us in ${ }^{1}$ ) to be successful, the actual cxistence of this risc is absolutely cssential and a knowledge of its magnitude important.

The theory of Landau and Sternheimer on the variation of the most probable energy loss as a function of $p /(m c)$ has been verified in a solid medium and found to be correct within $\pm 1 \%$ up to $p /(m c)=100$ and within $\pm 3 \% \%$ at $p /(m c) \approx 20000$ in a recent experiment by Bellamy et al. $\left.{ }^{6}\right)$. There are no experiments done, to our knowledge, of comparable accuracy with gaseous detectors with which category alone we are concerned. In the early experiments by Ghosh et al. ${ }^{7}$ ) and later by Kepler et al. ${ }^{8}$ ), the relativistic rise of probable

* Research supported by the National Science foundation and the U.S. Office of Naval Research, Contract Nonr 1224(23).

† One leave of absence from Tata Institute of Fundamental Research. Bombay-5, India. energy loss. For this method to be successful, it is important to verify experimentally the magnitude of the relativistic rise further up to values of $p /(m c) \approx 2000$. An experiment carried out to verify this is described and the results presented. The results seem to show that the experimentally determined relativistic rise of the most probable energy loss is less than what the theory predicts.

specific ionization (i.e. the number of ions occurring either singiy or in groups of less than a specified size along the trajectory of a particle in a unit length) was measured in various gases. Though this is not the quantity that is recorded in the gas proportional counters proposed by us, it is disquieting to note that the relativistic rise observed in these experiments was less than what the theory predicted at $p /(m c) \approx 100$ in several gases, the sole exception being helium [see also Ballario et al. $\left.\left.{ }^{9}\right)\right]$. The experiments ${ }^{10-13}$ ) in which gas proportional counters were employed to measure the relativistic rise of the most probable energy loss either do not extend $\left.{ }^{10}\right)$ to the range of $p /(m c)$ values of our interest or show ambiguous results ${ }^{11.12}$ ). These early experiments had to depend on cosmic radiation for the source of high energy particles as a result of which the momentum bites and the errors were large. The lack of experimental proof of the correctness of the theoretical estimates of the relativistic rise of the most probable energy losses in gaseous media in the range of $p /(m c) \gtrsim$ 100 and the existence of suggestion that the theory might be overestimating prompted us to undertake this investigation.

\section{Experiment}

The experimental arrangement is shown in fig. 1 which is self explanatory. The experiment was carried out at the bevatron at Lawrence Radiation Laboratory, Berkeley (U.S.A.) during January, 1968.

The proportional counter, from which pulses were analyzed for the most probable energy loss, was of $15 \times 15 \mathrm{~cm}^{2}$ square cross-section, $40 \mathrm{~cm}$ long and had a $0.013 \mathrm{~cm}$ diameter steel wire stretched at the center. The body of the counter was made up of $0.15 \mathrm{~cm}$ thick 


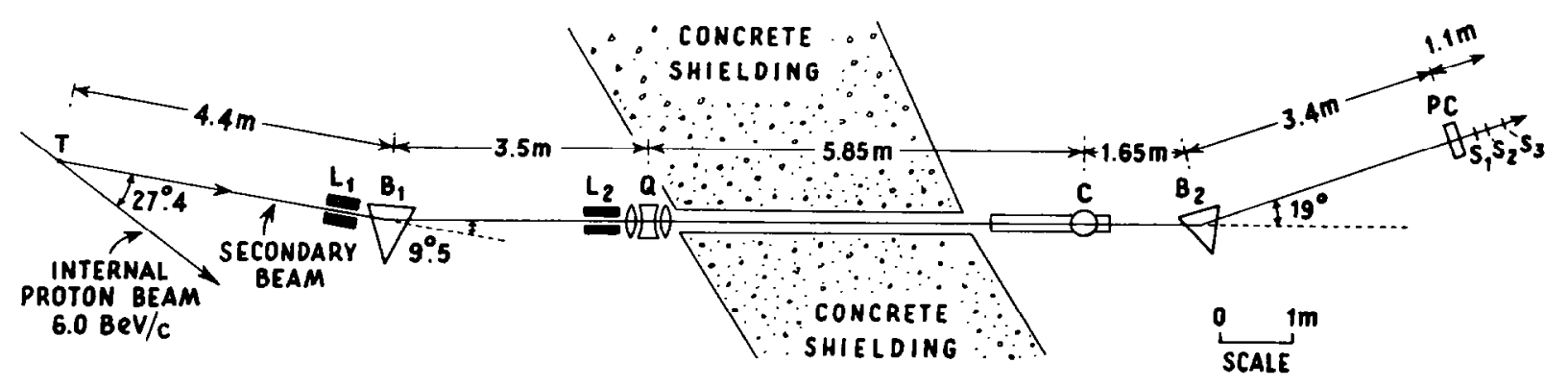

Fig. 1. Experimental arrangement.

$T$ Internal target

$\mathrm{L}_{1}, \mathrm{~L}_{2}$ Lead collimators (40 and $50 \mathrm{~cm}$ long)

$B_{1}, B_{2}$ Bending magnets $\left(90 \times 40 \times 20,90 \times 45 \times 20 \mathrm{~cm}^{3}\right)$

Q Focussing magnet

C Čerenkov counter $\left(\mathrm{CO}_{2}\right.$ gas)

PC Proportional counter $\left(40 \times 15 \times 15 \mathrm{~cm}^{3}\right)$

$\mathrm{S}_{1} \mathrm{~S}_{2} \mathrm{~S}_{33}$ Scintillation counters $\left(5 \times 5 \times 0.6,7.5 \times 7.5 \times 1.2,7 \times 7.5 \times 0.6 \mathrm{~cm}^{3}\right)$.

aluminum. There was a $12 \times 12 \mathrm{~cm}^{2}$ hole on one of the sides of the counter which was covered with a $0.0025 \mathrm{~cm}$ thick aluminum foil to serve as a window through which the charged particles entered the counter. The counter was continuously flushed with a gas mixture of argon (93\% by molecular concentration) and methane $(7 \%)$. The impurities in the gas due to $\mathrm{N}_{2}$, $\mathrm{O}_{2}$ and $\mathrm{H}_{2} \mathrm{O}$ were less than $30 \mathrm{ppm}$. The counter was operated at voltages $\approx 2800 \mathrm{~V}$. Gas multiplication values under these conditions of operation were $\approx 500$. Pulses from the proportional counter were amplified by a charge sensitive preamplifier and then by a double delay line amplifier before being fed to a pulse height analyzer for their analysis.

The gas Cerenkov counter is filled with carbon dioxide at various pressures up to 250 pounds per square inch depending upon the momentum of the particle beam. It is of $20 \mathrm{~cm}$ diameter circular crosssection and approximately $100 \mathrm{~cm}$ long. The Cerenkov light is viewed by two photomultipliers, A and B. Under a given set of experimental conditions like beam momentum, gas pressure, etc., one obtains the ratios of the coincidence rates $\mathrm{S}_{1} \mathrm{~S}_{2} \mathrm{~S}_{3} \mathrm{~A} / \mathrm{S}_{1} \mathrm{~S}_{2} \mathrm{~S}_{3}$, $\mathrm{S}_{1} \mathrm{~S}_{2} \mathrm{~S}_{3} \mathrm{~B} / \mathrm{S}_{1} \mathrm{~S}_{2} \mathrm{~S}_{3}$ and $\mathrm{S}_{1} \mathrm{~S}_{2} \mathrm{~S}_{3} \mathrm{AB} / \mathrm{S}_{1} \mathrm{~S}_{2} \mathrm{~S}_{3}$ where, for example, $S_{1} S_{2} S_{3} A$ is the coincidence rate between the scintillators $S_{1}, S_{2}$ and $S_{3}$ and the output pulses from the photomultiplier A of the Cerenkov counter. From these three ratios, it is possible to calculate the concentration of electrons in the beam as well as the absolute efficiencies $\varepsilon_{\mathrm{A}}$ and $\varepsilon_{\mathrm{B}}$ of the two photomultipliers viewing the Cerenkov counter. During the actual experiment, "Cerenkov counter output", $C$, is generated by feeding the two pulses from the two photomultipliers $A$ and B into an OR circuit. The efficiency of the Ceren- kov counter, $\varepsilon_{C}$, when operated in this fashion is experimentally determined to be $90^{\circ}{ }_{i}$.

One of two targets were used during the experiment. One is $1.2 \times 0.6 \times 5 \mathrm{~cm}^{3}$ copper and the other $0.15 \times$ $\times 0.6 \times 0.9 \mathrm{~cm}^{3}$ "heavymet", an alloy of tungsten and copper.

The magnitudes of currents in the two bending magnets defined the momentum of the particle beam, there being no other element in the experiment to measure the momentum. Changing the current in one of the magnets by $\pm 5_{i 0}^{\circ}$ from its calculated value to select particles of a given momentum while maintaining the current in the other at its calculated valuc decreased the $S_{1} S_{2} S_{3}$ coincidence rates almost always by a factor $\gtrsim 2$. This assured us that the momentum bite is $\approx \pm 5^{\circ}$, , quite adequate for our purposes since the energy loss is a weak function of particle momentum.

The experimental procedure was the following. When a particular value of the momentum of the particles is decided upon, currents in the bending magnets $B_{1}$ and $B_{2}$ necessary to transport a beam of this momentum are read off from the standard magnet calibration curves and are established. Pressure in the Cerenkov counter is adjusted such that the counter operated below the threshold for muons and pions but much above the threshold for electrons of this momentum. Coincidences $\mathrm{S}_{1} \mathrm{~S}_{2} \mathrm{~S}_{3} \mathrm{C}$ which are due to electrons in the beam opened the gate to 400 channel pulse height analyser which, then, analyzed the pulses from the proportional counter only due to electrons of this momentum. Keeping everything else the same, the coincidence requirement is changed to $\mathrm{S}_{1} \mathrm{~S}_{2} \mathrm{~S}_{3} \overline{\mathrm{C}}$ to generate the gate thus selecting pions and muons. The muons are believed to constitute a small pereentage 
( $\approx 5$ to $10 \%$ ) of pions*. Thus at each momentum, we had taken two runs one with electrons and the other with pions which provided us two values for the most probable energy loss at two widely separated values of $p /(m c)$. Throughout the experiment, only negatively charged particle beams are used. The intensity of the beam is adjusted at each momentum by choosing one of the two targets and manipulating their position inside the bevatron such that the proportional counter counted only $\leqslant 5000$ counts during the spill time of approximately $800 \mathrm{msec}$. The trigger rates, which were different at different momenta, were approximately $40 /$ spill in the case of pions and $5 /$ spill for electrons. There were 11 spills per minute. We analyzed 14000 to 25000 pulses due to electrons and a similar number due to pions at each momentum. The proportional counter was calibrated by X-rays of energies 22.2, 14.4 and $8.05 \mathrm{keV}$ before and after each run.

\section{Data analysis}

The quantity that is measured in our experiment is the magnitude of a pulse that is proportional to the number of ion pairs produced in the counter by an incident charged particle. To take this quantity as the energy loss suffered by the incident charged particle in the counter, the quantity discussed in theories, two assumptions have to be made:

1. The number of ion pairs is proportional to the energy loss;

2. No energy of the energy loss that actually took place in the counter leaks out of the counter nor is brought in from the energy losses occurring outside the gaseous region.

These assumptions are very reasonable and in what follows we regard them to be true.

The distribution in the energy losses, $g(\Delta)$, in the counter is more conveniently described in terms of a dimensionless parameter $A, f(A)$, where

$$
\Lambda=\left(\Delta_{\mathrm{mp}}-\Delta\right) / \xi \text {. }
$$

Here $\Delta_{\mathrm{mp}}$ is the most probable energy loss, $\Delta$ the energy loss in any arbitrary case and

$$
\xi=0.300 m c^{2}(Z / A) t / \beta^{2},
$$

where $m c^{2}$ is the rest mass of the electron; $t$, the counter gas thickness in $\mathrm{g} / \mathrm{cm}^{2}, Z$, the atomic number and $A$, the atomic weight of the medium (the counter gas) and $\beta c$ the velocity of the incident particle.

\footnotetext{
* Since the most probable energy loss varies with $p /(m c)$ very slowly and since, at any given momentum, the values of $p /\left(m_{n} c\right)$ and $p /\left(m_{\mu} c\right)$ are not widely different, it is safe to ignore the small contamination of the beam by muons.
}

We have tried to fit our data to two distributions, one, $f_{\mathrm{L}}(\Lambda)$, calculated by Börsch-Supan $\left.{ }^{14}\right)$ on the basis of the Landau theory ${ }^{2}$ ) and the other $f_{\mathrm{BL}}(\Lambda)$ given by Blunck and Liesegang ${ }^{15,16}$ ) who improved the Landau theory for very thin detectors by considering the details of binding effect of the electrons in the various atomic shells. These effects become important when the binding energy of the electron in the $K$ shell is not smaller than the most probable energy loss by at least two or three orders of magnitude. In our case, the most probable energy loss is in the range 22 to $36 \mathrm{keV}$ and the binding energy of the $\mathrm{K}$-shell electron in argon is $3.20 \mathrm{keV}$; hence Blunck and Liesegang modifications become important. We performed $\chi^{2}$ calculations in comparing the experimental distribution of energy losses with theoretical distributions for several values of $\Delta_{\mathrm{mp}}$ to obtain the best value of $\Delta_{\mathrm{mp}}$. We have shown in fig. 2a, b two typical pulse height distributions from our experiment together with the two theoretical dis-
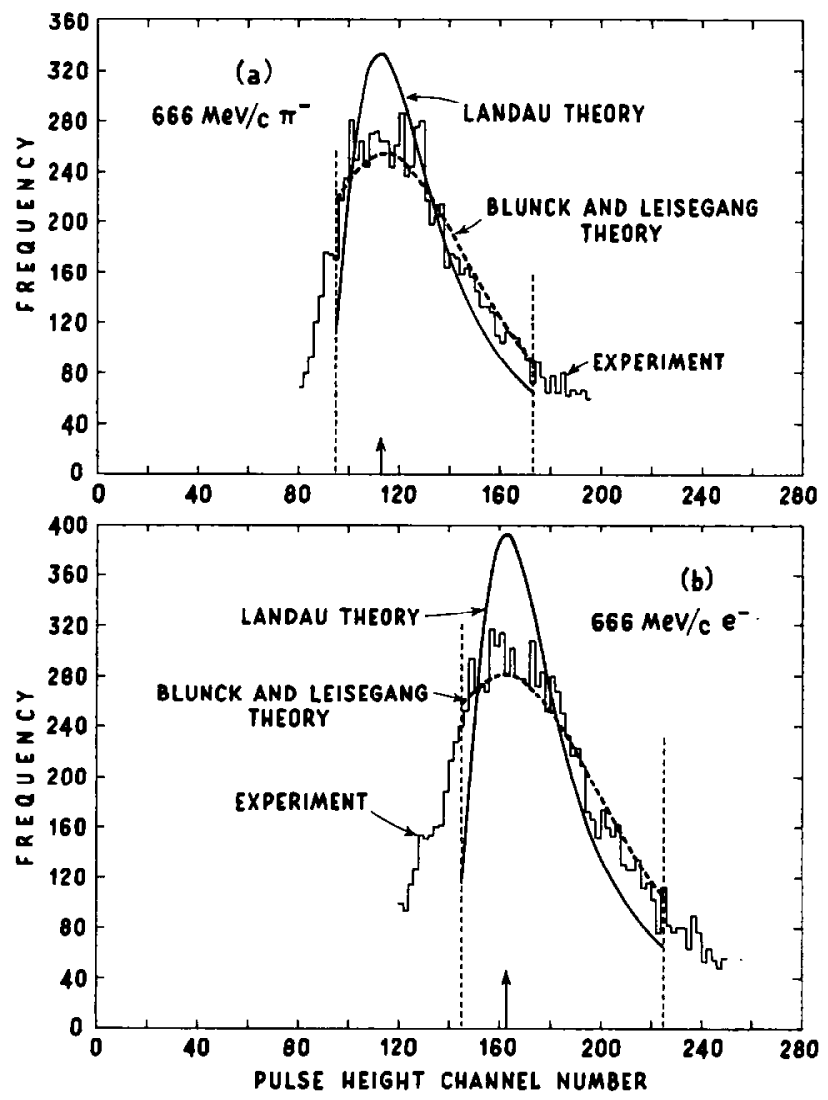

Fig. 2. Pulse height distribution of pulses from the proportional counter is shown when the incident particles are $666 \mathrm{MeV} / \mathrm{c}$ pions (a) and $666 \mathrm{MeV} / \mathrm{c}$ electrons (b). Also shown are the theoretical distributions due to Landau (calculations by Börsch-Supan) and due to Blunck and Liesegang normalized to the total number of events between the dotted lines. 


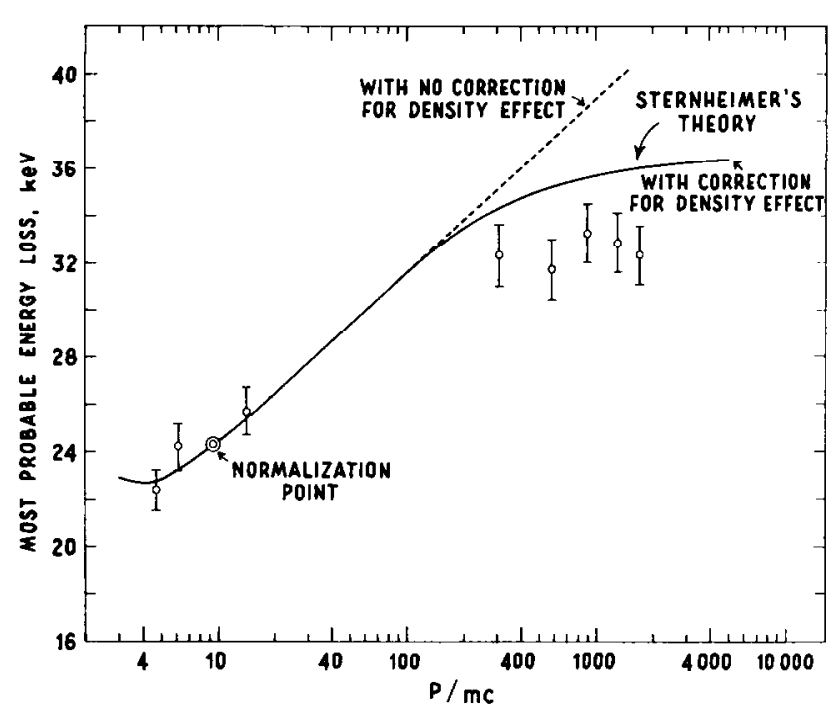

Fig. 3. Variation of the most probable energy loss with $p /(m c)$ of the incident beam. Points are from experiment and the curves are from theories of Landau and Sternheimer. Experimental data is nomalized with theory at $p /(m c)=9.5$.

tributions fitted to the data. As shown by us in greater detail in our previous work $^{1}$ ), we find now also that Blunck and Liesegang theory describes the experimental distributions better than Landau theory though not as well as one would hope. As examples, we find in the case of data presented in fig. $2 a, \chi_{\text {Landau }}^{2}=312$ and $\chi_{\mathrm{BL}}^{2}=108^{*}$ for 79 degrees of freedom. The location of the peak from the $\chi^{2}$ distribution is correct to within $\pm 2 \%$. The absolute values of $\Delta_{\mathrm{mp}}$ resulting from the $\chi^{2}$ analysis are about $20 \%$ lower than the theoretical predictions. The reason for this discrepancy is not known. We have, therefore, normalized the experimental data on the variation of the most probable energy loss with $p /(m c)$ with the theoretical value at $p /(m c)=9.5$.

\section{Results and discussion}

We have shown in fig. 3 the variation of the most probable energy loss as a function of $p /(m c)$. Also shown in the figure are the theoretical variations of the same as given by the expression ${ }^{2-4}$ )

$$
\Delta_{\mathrm{mp}}=\xi\left[\ln \left\{2 m c^{2} \xi / I^{2}\left(1-\beta^{2}\right)\right\}-\beta^{2}+0.37-\delta\right],
$$

where $I$ is the mean ionization potential of the medium, $\delta$ the correction due to density effect and all other symbols defined earlier. The curve labelled "with no

* In the actual computation of $\chi^{2}$, we varied both the values of $1 \mathrm{mp}$ and $\xi$ to find a minimum in $\chi^{2}$ using the Blunck- Liesegang theory. A slight distortion of $\xi$ has brought down the $\left(\chi_{\mathrm{BL}}\right)^{2}$ value to about 80 . correction for density effect" is the one calculated by putting $\delta=0$ in the above expression and the curve "with correction for density effect" by putting in the value of $\delta$ given by Sternheimer ${ }^{5}$ ). While calculating the theoretical variations, due weights are attached to the two constituents, argon and methane, of the medium. As stated earlier, the experimental data are normalized with theory at $p /(m c)=9.5$. The $p /(m c)$ values cluster in two groups, one at low values of $p /(m c)$ which is all due to pions and the other at high values all due to electrons. It was not possible to fill the gap in between in this experiment as there were too few pions at momenta $p \gtrsim 4.0 \mathrm{GeV} / c$ and too few electrons at momenta $p \leqslant 150 \mathrm{MeV} / c$. The errors shown in $Y$ coordinates of the points include those in locating the peak $\left( \pm 2^{\circ},{ }_{0}\right)$ and the reproducibility of the experiment. The errors in the $X$-coordinates of the points, not shown in the figure, are estimated to be $\pm 5 \%$ which are sufficiently small.

It is clear from fig. 3 that the experimentally determined values for the relativistic rise are smaller than what the theory predicts. The theory predicts an increase of $60^{\circ}$, from its value at the minimum to its value at $p /(m c) \approx 2000$ whereas the best "eys-fit" line to the experimental data indicates that the rise is only $45^{\circ}{ }_{0}^{\circ}$ with an error $\approx \pm 5^{\circ}{ }_{0}$.

At this stage, it is important to see if there is any experimental deficiency that could be responsible for the discrepancy. If a significant fraction of the "pion" triggers, namely $S_{1} S_{2} S_{3} \bar{C}$, are caused by electrons, then this would result in raising the measured most probable energy loss at low values of $p_{i}^{\prime}\left(m c^{\prime}\right)$ which would thus reduce the experimentally determined relativistic rise. This can only happen if the electrons in the beam fail to produce a signal output from the Cerenkov counter. The inefficiency of the Cerenkov counter is $\approx 10^{\circ}{ }_{0}$ and the concentration of the electrons in the beam is $\approx 10^{\circ}{ }_{0}$; these two figures imply that $\approx 1^{\circ}{ }_{0}^{\circ}$ of the triggers could have been caused by electrons. This value is small enough to rule out the electrons imitating pions in their signature to be the reason for the discrepancy. It is also conceivable that a significant fraction of "electron" triggers, $\mathrm{S}_{1} \mathrm{~S}_{2} \mathrm{~S}_{3} \mathrm{C}$, are caused by pions; this would, then, lower the recorded most probable energy loss at high values of $p(m c)$ thus explaining the discrepancy. Such a thing can happen:

1. Due to chance coincidences between $\mathrm{S}_{1} \mathrm{~S}_{2} \mathrm{~S}_{3}$ and $C$;

2. Due to production in the entry wall $\left(0.48 / \mathrm{cm}^{2}\right.$ of aluminum) or in the gas $\left(\approx 1.7 \mathrm{~g} / \mathrm{cm}^{2}\right.$ of $\left.\mathrm{CO}_{2}\right)$ of the Čerenkov counter of a knock-on electron with sufficient energy, correct direction with respect to the beam and enough path length to produce a Cerenkov 
signal. The observations on the counting rates show that the chance coincidence rates are always $\leqslant 3^{\circ}{ }_{0}^{\circ}$ of the genuine triggers. It is calculated that $3^{\circ}{ }_{0}$ of the "electron" triggers could have been produced by the pions producing a $\delta$-ray in the Čerenkov counter. Such small contaminations, $\leqslant 6^{\circ}{ }_{i}$, could not have shifted (towards lower values) the peak in the energy loss distribution by electrons by more than 1 or $2^{\circ}$, whereas the discrepancy seems to be $\approx(15 \pm 5)^{\circ}{ }_{0}$.

It is known that part of the relativistic rise in the energy loss in the gas of the proportional counter is in the form of Cerenkov radiation. It is conceivable that a part or whole of this radiation does not contribute to ionization which is actually measured in the experiment. This can explain the discrepancy if its magnitude is sufficiently large. Sternheimer ${ }^{5}$ ), however, calculated that the energy loss in the form of Cerenkov radiation that would not ionize the gas in the vicinity is $\leqslant 0.017 \mathrm{MeV} /\left(\mathrm{g}^{\prime} \mathrm{cm}^{2}\right)$ in all the gases heavier than helium. This corresponds to $\leqslant 0.40 \mathrm{keV}$ of energy loss which constitutes $\leqslant 3^{\circ}$ o of the relativistic rise.

Since the effects discussed are small and we do not see any other effects that could be responsible, the observed discrepancy. $\approx(15 \pm 5)^{\circ}$, seems to be genuine within the significance of the errors.

We cannot exactly point out the reason for the discrepancy. Some of the possible sources are

1. The variation of the most probable energy loss with $p /(m c)$ as given by Landau ${ }^{2-4}$ ) (curve marked "with no correction for density effect" in fig. 3) might be incorrect, or

2. The magnitude of the correction for the density effect (difference between the two curves in fig. 3) due to Sternheimer ${ }^{5}$ ) might be incorrect.

These two aspects were verified and found to be accurate within $\pm 1^{0}{ }_{10}^{\circ}$ in the solids ${ }^{6}$ ). The reason why the theory should hold good in solids (of thickness $\gtrsim 0.5 / \mathrm{cm}^{2}$ ) and fail in gases (of thicknesses $\approx 0.025 \mathrm{~g} /$ $\mathrm{cm}^{2}$ ) is not obvious. For one thing. when the detector is of gas, it is very thin $\left(0.025 \mathrm{~cm}^{2}\right)$ and perhaps the same atomic binding effects as were responsible for the broadening of "Landau" distribution might be playing a role in determining the magnitude of the most probable energy loss.

\section{Conclusion}

The rise of the most probable energy loss with $p /(m c)$ as determined in the present experiment seems to be lower than what the theory predicts as shown in fig. 3 . We cannot say from this experiment whether

1. The discrepancy is distributed throughout the range of values of $p /(m c)$ i.e., the actual relativistic rise being only $\frac{3}{4}$ of the value estimated by the theory at all values of $p /(m c)$, or

2. The actual relativistic rise agrees with the theory up to $p /(m c) \approx 100$ and then the discrepancy starts appearing. If the true picture is according to (1.), the difference in the most probable energy losses by $100 \mathrm{GeV}$ pions and by protons of the same energy is only $7 \%$ instead of $10 \%$ assumed by us in ') while discussing the problem of distinguishing protons from pions in the cosmic radiation at energies $\gtrsim 100 \mathrm{GeV}$ on the basis of relativistic rise of the most probable energy loss. The pion-proton identification problem at energies $\gtrsim 100 \mathrm{GeV}$ is rendered therefore more difficult than that indicated in '). If the true picture, on the other hand, is according to (2), the pion-proton identification on the basis of relativistic rise is almost impossible and one has to reconsider the threshold Cerenkov method. The existence or otherwise of the relativistic rise at the range of values of $p /(m c)>100$ is an important point to be settled for evolving a practicable pion-proton separation method. While the present experiment indicates that the relativistic rise might be lower than the theoretical estimate, a more precise experiment, particularly at more evenly spaced values of $p /(m c)$, would be desirable.

The author is grateful to Professor L. W. Jones for many useful discussions and comments on a preliminary draft of this paper, and thanks E. Parker and S. Wilson for tending the apparatus while the various runs were being taken.

The author wishes to express his gratitude to Dr. B. Cork for providing the support and facilities at the Lawrence Radiation Laboratory and comments on the preliminary draft, the Lawrence Radiation Laboratory for hospitality and the operating crew of the bevatron for their cooperation.

The author would like to express his thanks to the University of Michigan Institute of Science and Technology for partial support of the experiment.

Note added in proof: Based on comments in a private communication from Professor R. M. Sternheimer, the following additional information might be useful.

1. The proportional counter was operated at atmospheric pressure at $900 \mathrm{ft}$ above sealevel. The density of the argon-methane gas mixture is calculated to be $1.543 \times 10^{-3} \mathrm{~g} / \mathrm{cm}^{3}$.

2. In the computations of the theoretical curves in fig. 2, I had used $I$ (argon) $=16.8$ Ry and $I$ (argon + methane $)=16.8 \mathrm{Ry}$, whereas Sternheimer recommended the values 15.45 and $14.51 \mathrm{Ry}$, respectively on the basis of his empirical formula (6) that appeared in 
Phys. Rev. 145 (1966) 247. In the same paper, Sternheimer also outlined the interpolation procedure to be adopted while calculating the density effect.

The changes to the theoretical curves in fig. 2 resulting from Sternheimer's suggestions are $\approx 1.5^{\circ}{ }_{0}^{\prime}$ in absolute magnitude and negligible (much less than $1.5^{0} \%$ ) when the experimental data are normalized with the theoretical curves at one of the points.

The author expresses his thanks to Professor R. M. Sternheimer for his comments.

\section{References}

1) P. V. Ramana Murthy and G. D. DeMeester, Nucl. Inst r. and Meth. 56 (1967) 93.

2) L. Landau, J. Exp. Phys. (USSR) 8 (1944) 201.

3) B. Rossi, High-energy particles (Prentice-Hall, 1952) p. 32.

4) R. M. Sternheimer, Phys. Rev. 91 (1953) 256.

5) R. M. Sternheimer, Phys. Rev. 103 (1956) 511.
6) E. H. Bellamy, R. Hofstadter, W. Lakin, J. Cox, M. Perl, W. Toner and T. F. Zipf, Phys. Rev. 164 (1967) 417.

7) S. K. Ghosh, G. M. B. Jones and J. G. Wilson, Proc. Phys. Soc. (London) 65A (1952) 68; 67A (1954) 331

$\left.{ }^{8}\right)$ R. G. Kepler, C. A. D'Andlau, W. B. Fretter and L. F. Hansen, Nuovo Cimento 1 (1958) 71.

9) C. Ballario, A. De Marco, R. D. Fortune and C. Verkerk, Nuovo Cimento 19 (1961) 1142.

10) J. Becker, P. Chanson, E. Nageotte. P. Trille, B. T. Price and P. Rothwell, Proc. Phys. Soc. 65A (1952) 437.

11) J. K. Parry, H. D. Rathgeber and J. L. Rouse, Proc. Phys. Soc. 66A (1953) 541.

12) E. D. Palmatier, J. T. Meers and C. M. Askey, Phys. Rev. 97 (1955) 486.

13) For more references on this subject, R. M. Sternheimer, Methods of experimental physics 5A (Academic Press, New York, 1961) Ch. 1-1.

14) W. Börsch-Supan, J. Res. National Bureau of Standards 65B (1961) 245

15) O. Blunck and S. Liesegang, Z. Physik 128 (1950) 500.

16) O. Blunck and K. Westphal, Z. Physik 130 (1951) 641. 